\title{
EDITORIAL
}

\section{Recent Developments in the Application of Polymeric Nanoparticles as Drug Carriers}

${ }^{1}$ Department of General and Analytical Chemistry, Faculty of Chemical Technology, Institute of Chemistry and Technical Electrochemistry, Poznan University of Technology, Poland

${ }^{2}$ NanoBioMedical Centre, Adam Mickiewicz University, Poznań, Poland

A - research concept and design; B - collection and/or assembly of data; C - data analysis and interpretation;

$\mathbf{D}$ - writing the article; $\mathbf{E}$ - critical revision of the article; $\mathbf{F}$ - final approval of article

\begin{abstract}
Nanotechnology is an interdisciplinary field of science offering interesting solutions for many branches of human life. Nanomaterials, defined as structures with at least one dimension below $100 \mathrm{~nm}$, are the focus of increasing research attention as versatile tools for nanomedicine. Among the various nanostructures recently described in the literature, polymeric nanoparticles, characterized by satisfying biocompatibility, have aroused great interest as the carriers for various biologically active substances such as drugs, proteins and nucleic acids. These nanoparticles have already been reported as efficient vehicles for therapeutic agents in many disease entities. They can be delivered to the body via different administration routes. This review addresses recent advances in the usage of polymeric nanoparticles as drug carriers described in the years 2013 and 2014. The advantages of polymeric nanocarriers for medical application are highlighted, including their low toxicity, evaluated in vitro and in vivo. Moreover, the classification of polymeric nanoparticles is presented as well as various protocols of their synthesis (Adv Clin Exp Med 2015, 24, 5, 749-758).
\end{abstract}

Key words: biocompatibility, polymeric nanoparticles, drug carrier, nanomedicine.

The enormous progress recently observed in the field of nanotechnology provides many interesting tools for medical sciences. Nanomaterials are structures with at least one dimension below $100 \mathrm{~nm}$ [1]. These materials possess unique features such as large surface-to-volume ratio resulting from their small dimensions. The specific characteristics of nanostructures make them extremely attractive as valuable devices for nanomedicine. Among the various nanomaterials, quantum dots [2], graphene [3], carbon nanotubes [4], metallic and metal oxide nanoparticles [5] and polymeric nanoparticles [6] have already been reported as promising candidates for medical applications. Particularly the latter have recently been investigated as carriers for biologically active substances and widely used for the potential treatment of many disease entities.

Polymeric nanoparticles may provide targeted drug delivery, especially desirable for cancer therapy, markedly decreasing the systemic side effects of highly toxic anticancer drugs [7]. Moreover, the polymer-based nanocarriers may enhance the bioavailability of poorly water-soluble drugs and provide their sustained release $[8,9]$. The numerous advantages offered by polymeric nanoparticles as drug delivery systems (DDSs) also include the extension of drug circulation time in the body [10], increased stability of acid-labile drugs [11], uniform drug distribution in the targeted site e.g. alveoli [12], high drug encapsulation efficiency [13] and stimuli-responsiveness e.g. to oxidative stress [14] or hypoxia [15]. Furthermore, polymer-based nanocarriers provide high thermodynamic stability to the system [15] and can easily permeate through various biological barriers [16]. It deserves to be emphasized that surface modification of polymeric nanoparticles can markedly increase the interaction of active substance with the biological target [17]. Intriguingly, the encapsulation or dispersion of drugs with a polymeric matrix might protect the drug molecules from 
premature release and degradation before reaching the targeted site [18]. Polymer-based nanoparticles can be used as the carrier for numerous biologically active molecules including drugs [18], proteins [7], monoclonal antibodies [13], nucleic acids $[19,20]$, biological extracts $[16,21]$ and others [22]. This review will be especially concerned with the application of polymer-based nanoparticles for the treatment of various disease entities including bacterial [23, 24], fungal [25] and parasitic [26] infections, ulcers [11], hypertension [12], angina [8], glaucoma [9], uveitis [27], asthma [22], cancer $[15,28]$, neurodegenerative disease [29] and many others $[30,31]$. It deserves to be emphasized that several polymers are characterized by satisfying biocompatibility and predictable biodegradability $[9,22]$ while others are non-degradable or undergo slow degradation [22, 32]. Remarkably, polymer-based nanoparticles can be administered orally $[20,23]$, intravenously [18], percutaneously [30], ophthalmically [27], pulmonarily [12, 25], transmucosally to nose and lungs [33] or delivered to the brain via inner ear administration [16]. It is noteworthy that selected polymers are approved by the Food and Drug Administration (FDA) to be used for therapeutic applications [27].

\section{Classification and Properties of Polymeric Nanoparticles}

Recent advances in nanotechnology provide us with a wide range of polymers able to be formulated into nanoparticulate carriers for various active substances. Numerous synthetic and natural polymeric materials, including biodegradable, biocompatible and non-biodegradable substances, have already been used for nanoparticle preparation. Among the synthetic biodegradable polymeric matrices, poly(lactide-co-glycolide) (PLGA) $[26,28,34]$ and poly(D, L-lactide) (PLA) [35] are the most common. Polymeric materials such as poly(methylmethacrylate) (PMMA) [36] and poly(ethylene-co-vinyl acetate) (PEVA) [12] are characterized by satisfying biocompatibility. On the other hand, poly(ethylene glycol) (PEG) [15] and Eudragits ${ }^{\circledR}[10,31]$ belong to the group of nonbiodegradable synthetic polymeric materials. The -biodegradable natural polymers already used for the preparation of nanoparticulate DDSs include poly(L-glutamic acid) (PGA) produced by Bacillus subtilis [37], pullulan produced from starch by the fungus Aureobasidium pullulans [33], gelatin [6], alginate [9, 33], chitosan [15] and its derivatives including N-palmitoyl chitosan [14] or mannosemodified trimethyl chitosan-cysteine (MTC) conjugate [20] and many others [19].
Intriguingly, the widespread application of PLGA results from its relatively inert composition, stable rate of degradation and known degradation products [22], namely lactic acid and glycolic acid [23]. PLGA-based nanoparticles are characterized by good mechanical stability and narrow size distribution [38]. This polymer, used as a matrix during nanoparticulate carrier preparation, can protect the drugs from enzymatic degradation [27] after oral administration [23]. More relevantly, it has been reported that the drug release rate can be controlled by the PLGA molecular weight as well as by the glycolide to lactide ratio [23]. Polymerbased nanoparticles are also promising candidates for non-invasive transdermal delivery without removing the stratum corneum [30]. In this view, especially biocompatible and biodegradable chitosan possessing mucoadhesive properties can significantly enhance penetration into the deeper skin layers [30]. Furthermore, similarly to PLGA, it has the approval of the FDA for biomedical usage [15, 22]. Remarkably, PEG can be used as a stabilizer during polymeric nanoparticle preparation [15] or applied as a linker for conjugation of targeting ligands to the surface of the nanoparticles [17]. Additionally, the modification of drug carriers with water-soluble PEG can contribute to a significant increase of the circulation half-life in vivo of asprepared nanovehicles [18].

Biologically active substances can be encapsulated in polymeric nanoparticles and consist of a so-called core-shell system [34]. In such a case, an active agent is protected from degradation by a polymeric coat able to be functionalized to specifically recognize the targeted site [28]. Other strategies consist of dispersion of the active substance in the polymeric matrix [24]. Recently, the polymeric form of a prodrug containing the molecules of the active substance coupled with the polymeric backbone has been demonstrated to release the therapeutic agent upon hydrolytic degradation [39]. Curiously, drug-loaded polymeric nanoparticles incorporated into other matrices (e.g. synthetic polymers) can consist of a component of materials designed for biomedical applications [35].

\section{Synthesis of Polymeric Nanoparticles}

Polymeric nanoparticles can be fabricated using various synthesis techniques. Nanoprecipitation, emulsion/solvent evaporation, emulsification, desolvation and polyelectrolyte complexation can be listed among the numerous synthesis approaches, and will be exemplified below. It is worth mentioning that the most common methods of 
polymer-based nanoparticle preparation are nanoprecipitation, emulsion/solvent evaporation and its modifications. These approaches of polymeric nanoparticle synthesis are schematically presented in Fig. 1. In the next paragraph, the strategies most recently described demonstrating fabrication of polymeric nanoparticles will be presented. Some examples are reviewed in the main text while others are summarized in Table 1.

Zhao and Feng [13] have described the preparation of nanoparticles composed of poly(lactide)-D-a-tocopheryl polyethylene glycol 1000 succinate copolymer (PLA-TPGS) and carboxyl groupterminated TPGS (TPGS-COOH) using the nanoprecipitation method. The previously synthesized polymers, PLA-TPGS and TPGS-COOH, and the drug were dissolved in acetone and dropwise added to water to form nanoparticles. The as-prepared carrier was loaded with docetaxel and chemically conjugated with a humanized monoclonal antibody via a carboxyl terminal group present in the prepared polymeric matrix [13]. A curious instance of carvedilol-loaded nanoparticles composed of poly(ethylene-co-vinyl acetate) (PEVA) prepared via the emulsion/solvent evaporation method has been described by Varshosaz et al. [12]. In this work, the solution of the polymer in carbon tetrachloride was mixed with a solution of the drug and PEG400 in dichloromethane. The as-prepared mixture was dispersed in the aqueous phase containing Tween 20 and agitated until complete evaporation of the organic phase [12]. Interesting studies have been also conducted by Alai and Lin [11] who have prepared lansoprazole-loaded polymeric nanoparticles composed of PLGA. The nanoparticles were prepared using a double water-in-oil-in-water (W/O/W) emulsion/solvent evaporation method. The drug and polymer dissolved in a mixture of dichloromethane and acetone were emulsified with an aqueous solution of $\mathrm{NaHCO}_{3}$. The as-prepared primary emulsion was added to the aqueous solution of PVA and emulsified. After stirring, the emulsion was evaporated and the nanoparticles were collected after centrifugation [11]. It is also worth mentioning that the group of Hazra et al. [36] have used an oil-in-water $(\mathrm{O} / \mathrm{W})$ modified atomized emulsification process to fabricate core/shell poly(methyl methacrylate) (PMMA)/biosurfactant nanoparticles. The nanoparticles were loaded with ibuprofen, anthraquinone and curcumin. As biosurfactants, rhamnolipid, surfactin and trehalose lipid have been

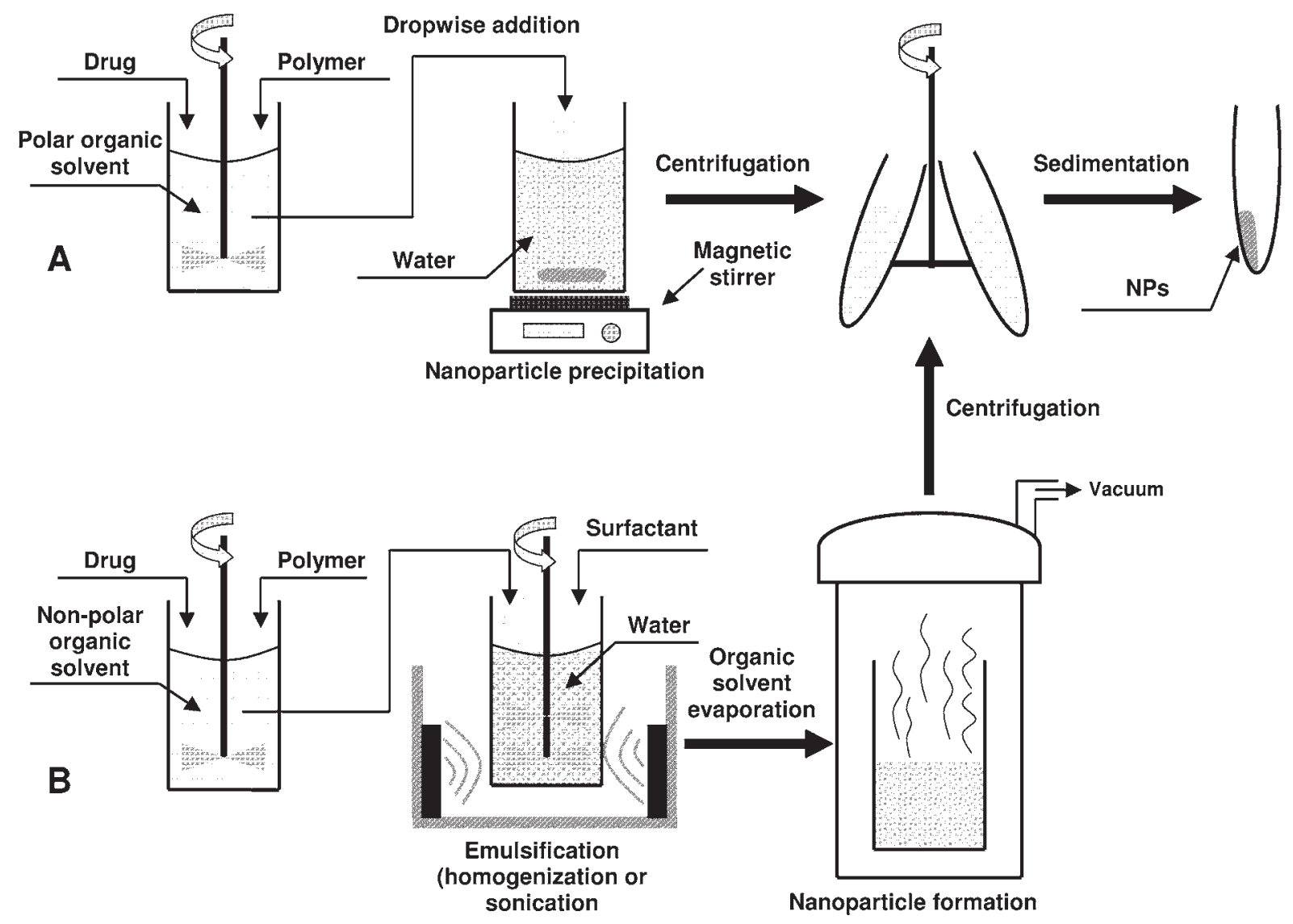

Fig. 1. Two approaches of polymeric nanoparticle preparation: (A) precipitation, (B) emulsification and solvent evaporation 
Table 1. Various strategies of polymeric nanoparticle fabrication

\begin{tabular}{|c|c|c|c|}
\hline Technique & Polymeric matrix & Active agent & Reference \\
\hline \multirow[t]{3}{*}{ Nanoprecipitation } & PLGA, TPGS & docetaxel & {$[17]$} \\
\hline & PLGA & cefixime & {$[24]$} \\
\hline & PLGA & felodipine & {$[8]$} \\
\hline Emulsification & POX & hydroxybenzyl alcohol & {$[22]$} \\
\hline \multirow{4}{*}{$\begin{array}{l}\text { Emulsification and solvent evapora- } \\
\text { tion }\end{array}$} & PLGA & cinnamon bark extract & {$[21]$} \\
\hline & PLGA & curcumin & {$[28]$} \\
\hline & PLGA & $\begin{array}{l}\text { salvianolic acid } B \text {, tanshinone } \\
\text { IIA, panax notoginsenoside }\end{array}$ & {$[16]$} \\
\hline & PLGA & vancomycin & {$[23]$} \\
\hline Emulsification or nanoprecipitation & gelatin, PLA & doxorubicin & {$[6]$} \\
\hline Multiple emulsification & PLGA & voriconazole & {$[25]$} \\
\hline Free radical emulsion polymerization & PMMA & TO-PRO3 & {$[38]$} \\
\hline Ionic cross-linking & chitosan & $\begin{array}{l}\text { hydrocortisone, hydroxyty- } \\
\text { rosol }\end{array}$ & {$[30]$} \\
\hline Electrospraying & Eudragit $^{\circledR}$ L100-55, Eudragit ${ }^{\circledR}$ RS PO & aspirin & {$[31]$} \\
\hline
\end{tabular}

CA - cholic acid; PEG - poly(ethylene glycol); PLA - poly(lactide); PLGA - poly(lactide-co-glycolide);

PMMA - poly(methyl methacrylate); POX - polyoxalate; TPGS D-a-tocopheryl polyethylene glycol 1000 succinate.

used. At the outset, the tenside was dissolved in water. Then, an aqueous solution of ammonium persulfate was added to the as-prepared solution to initiate the formation of free radicals. The monomer was sprayed over the surface of the biosurfactant solution to form nanoparticles [36]. As has been demonstrated by Katiyar et al. [9], the gelation method can be also employed for the preparation of polymeric nanoparticles. In this study, chitosan nanoparticles loaded with dorzolamide were formed by the dropwise addition of tripolyphosphate to a dorzolamide-containing chitosan solution. Finally, the sedimented nanoparticles were suspended in a sodium alginate solution to obtain an in situ gel [9]. A curious instance has been described by Narayanan et al. [18] who have used the desolvation method for preparation of poly(ethylene glycol)-modified gelatin nanoparticles loaded with ibuprofen. High molecular weight aggregates of gelatin dispersed in water were mixed with a solution of ibuprofen in ethanol. The dropwise addition of acetone resulted in precipitation of the ibuprofen-loaded nanoparticles which were subsequently cross-linked by adding calcium chloride [18]. Another group has prepared pullulan-based nanoparticles using the polyelectrolyte complexation method [33]. The negatively-charged sulfated and positively-charged aminated derivatives of the polymer were firstly synthesized by sulfonation and alkylation of the original polysaccharide, respectively. The interactions of sulfated pullulan-chitosan and aminated pullulan-carrageenan resulted in the formation of nanoparticles. The as-obtained nanocarriers were able to associate with the model protein, bovine serum albumin (BSA), demonstrating the potential of the as-prepared system for protein delivery [33].

The group of Kwon et al. [39] has conducted compelling studies on the preparation of nanoparticles based on a polymeric prodrug of vanillin. In this work, the polymeric material was composed of poly(vanillin oxalate) (PVO) covalently coupled vanillin in its backbone. The as-synthesized PVO dissolved in dichloromethane was added to the solution of poly(vinyl alcohol) (PVA) to form oil-in-water $(\mathrm{O} / \mathrm{W})$ emulsion. The nanoparticles were obtained by the evaporation of the solvent. The vanillin was released from the as-prepared nano-carrier upon its hydrolytic degradation [39].

\section{Toxicity and Bio- -compatibility Concerns}

The biocompatibility of polymeric nanoparticles used as a drug carrier is of crucial importance for development of drug delivery systems based on polymeric materials [33]. Hence, polymer-based 
nanoparticles should be examined through various in vitro and in vivo assays including cell viability, hemocompatibility and histological analysis [18]. One of the most common methods used to examine the in vitro cytotoxicity of polymeric nanoparticles is the MTT test [13]. In the MTT (3-(4,5-dimethylthiazol-2-yl)-2,5-diphenyl tetrazolium bromide) assay, the metabolic activity of cells is assessed relying on the evaluation of cellular enzyme activity [33]. In this test, the formation of blue formazan dye is related to mitochondrial dehydrogenase activity. High cell viability is ascribed to a high concentration of formazan dye, mirroring the high amount of metabolically active cells [33].

The group of Dionísio et al. [33] has examined the in vitro cytotoxicity of pullulan-based nanoparticles using MTT assay. The experiment was performed on respiratory Calu-3 cells, which is an immortalized cell line derived from lung adenocarcinoma. It has been found that upon $24 \mathrm{~h}$ exposure to the polymeric nanoparticle formulation, cell viability was in the range from 74 to $94 \%$ independently of polymer modification, concentration or incubation time. Such results indicate the biologically acceptable viability of Calu-3 cells without disturbing their metabolic functions when in contact with pullulan-based nanoparticles [33]. The MTT assay was also employed for the evaluation the cytotoxicity of poly(vanillin oxalate) (PVO) nanoparticles [39]. The test, performed on RAW 264.7 cells, revealed dose-dependent cytotoxicity after $24 \mathrm{~h}$ of incubation with PVO nanoparticles. Interestingly, no or a negligible cytotoxic effect was observed at nanoparticle doses less than $500 \mu \mathrm{g} / \mathrm{mL}$, indicating a satisfying biocompatibility of the prepared nanocarrier. Furthermore, tissue compatibility was examined using $\mathrm{Balb} / \mathrm{c}$ mice intramuscularly injected with $100 \mu \mathrm{L}$ of nanoparticles $(1 \mathrm{mg} / \mathrm{mL})$ suspended in phosphate buffer saline (PBS). The histological analysis of the mice, sacrificed at 7 days after injection, revealed minimal inflammatory response as demonstrated by the very little formation of immune and inflammatory cells [39]. Another group [36] has examined the in vitro cytotoxicity of core/shell poly(methyl methacrylate)/biosurfactant nanoparticles upon incubation with peripheral blood mononuclear cells using MTT assay. Moreover, the nanoparticle samples were exposed to anticoagulated human blood to assess the antihemolytic activity. The results of the MTT test revealed that cell survival decreased with increasing concentrations of the nanoparticles. Meanwhile, a hemolysis assay showed that nanoparticles coated with surfactin are less toxic than those surrounded by rhamnolipid and trehalose lipid biosurfactants [36].
Interestingly, Papa and co-workers [38] used a CellTiter96 ${ }^{\circledR}$ Aqueous Non-Radioactive Cell Proliferation Assay (3-(4,5-dimethylthiazol-2-yl)-5-(3-carboxymethoxyphenyl)-2-(4-sulfophenyl)-2H-tetrazolium, inner salt, MTS) to determine the cell viability of microglial cells treated with poly(methyl methacrylate) nanoparticles. Upon incubation with polymeric nanoparticles up to 6 days, the cellular viability was not affected [38].

As will be argued below, a wide range of other methods exist which are suitable for the evaluation of polymeric nanoparticles biocompatibility. To take one example, Yoo et al. [22] have studied lung biocompatibility after injection of a hydroxybenzyl alcohol-incorporated polyoxalate nanoparticle suspension to the trachea of Balb/c mice. Histological analysis of the lung revealed minimal inflammatory response and no changes of airway structure [22]. To take another example, Katiyar and co-workers [9] have shown the non-irritant properties of dorzolamide-loaded chitosan nanoparticles designed for the potential treatment of glaucoma. For this purpose, the ocular tolerance assay was employed, relying on the observation of adverse changes (e.g. hemorrhage, hyperemia and coagulation) that occur in the chorioallantoic membrane of the egg after exposure to the test material [9]. A curious instance has also been described by Narayanan et al. [18] who have examined the hemocompatibility of ibuprofen-loaded gelatin nanoparticles with or without modification with poly(ethylene glycol) (PEG). Interestingly, both kinds of nanoparticles did not induce any hemolysis up to $1 \mathrm{mg} / \mathrm{mL}$ and did not prolong coagulation times for particles in the concentration range from 0.1 to $1 \mathrm{mg} / \mathrm{mL}$. Moreover, in vitro experiments revealed that, upon exposure of peripheral blood mononuclear cells to nanoparticles, an insignificant immune response expressed by cytokine induction level, lymphocyte proliferation and suppression and lymphocyte toxicity was observed. The authors [18] have also examined in vivo toxicity after intravenous injection of nanoparticulate samples to Sprague Dawley rats. Pharmacokinetic studies revealed a prolonged drug release from the nanogelatin matrix and confirmed increased drug bioavailability from PEGylated vehicles. The improved bioavailability is ascribed to the hydrophilic nature of PEG molecules, providing a steric barrier able to decrease the adsorption of protein and enhance the circulation time in the body. The inflammatory response after a nanoparticle administration dose of $2.5 \mathrm{mg} / \mathrm{kg}$ revealed insignificant activation of the pro-inflammatory (INF- $\gamma$ and TNF- $\alpha$ ) and anti-inflammatory (IL-10, IL-4) cytokines. Histopathological analysis showed no apparent damage of tissue sections from the liver and kidney [18]. 
It is worth noting that in cancer therapy the toxicity driven by a nanoparticulate carrier has a desirable effect. For example, Kulhari and co-workers [7] employed the MTT test to assess the in vitro cytotoxicity of PLGA nanoparticles loaded with docetaxel and conjugated with bombesin peptide. The cellular toxicity studies using MDA-MB-231 breast cancer cells overexpressing gastrin releasing peptide (GRP) receptor revealed 12-fold higher toxicity of the as-prepared nanoparticles as compared to pure docetaxel and Taxotere. Interestingly, the toxicity of bombesin peptide-conjugated nanoparticles was significantly improved as compared to the docetaxel-loaded formulation in a dose-dependent manner from 6.25 to $100 \mathrm{ng} / \mathrm{mL}$. The results demonstrated the potential of a nanocarrier in anticancer therapy especially for the targeted therapy of cancer cells overexpressing GRP receptor [7]. Important studies have been also conducted by Zhao and Feng [13] who have employed the MTT test for an examination of the in vitro cytotoxicity of Herceptin-functionalized nanoparticles composed of poly(lactide)-D-a-tocopheryl polyethylene glycol 1000 succinate copolymer (PLA-TPGS) and loaded with doxorubicin. The viability studies were conducted using SK-BR-3 breast cancer cells upon exposure to the drug-loaded nanocarrier with or without functionalization with herceptin. The experiment revealed dose-dependent toxicity and a reduction of cellular viability upon incubation with antibody-functionalized nanoparticles as compared to a non-conjugated polymeric carrier. This may result from the intrinsic toxicity of Herceptin [13].

Other examples of cytotoxicity assays performed on cells exposed to polymer-based nanoparticles are summarized in Table 2.

Table 2. In vitro methods for evaluation of polymeric nanoparticle cytotoxicity

\begin{tabular}{|c|c|c|c|c|}
\hline Assay & Polymeric nanoparticles & Cell line & Comments & Reference \\
\hline MTT & $\begin{array}{l}\text { Eudragit }{ }^{\circledR} \text { NPs loaded with } \\
\text { aspirin }\end{array}$ & Caco-2 & $\begin{array}{l}\text { cell viability ca. } 65 \% \text { after } 48 \mathrm{~h} \text { of incuba- } \\
\text { tion }\end{array}$ & {$[31]$} \\
\hline MTT & $\begin{array}{l}\text { PLGA NPs loaded with silver } \\
\text { NPs and ascorbic acid }\end{array}$ & HepG2 & $\begin{array}{l}\text { not affected cells viability after } 24 \text { h of } \\
\text { incubation }\end{array}$ & {$[37]$} \\
\hline MTT & $\begin{array}{l}\text { Carboxymethyl dextran NPs } \\
\text { loaded with doxorubicin }\end{array}$ & SCC7 & $\begin{array}{l}\text { higher cytotoxicity of DOX-loaded NPs to } \\
\text { hypoxic than normoxic cells. Insignificant } \\
\text { cytotoxicity of unloaded NPs }\end{array}$ & {$[15]$} \\
\hline MTT & $\begin{array}{l}\text { NPs composed of chitosan } \\
\text { derivative loaded with curcumin }\end{array}$ & RAW 264.7 & $\begin{array}{l}\text { insignificant cytotoxicity for cells treated } \\
\text { with parent and curcumin-loaded NPs }\end{array}$ & {$[14]$} \\
\hline MTS & Polycationic NPs & $\begin{array}{l}\text { HEK293T } \\
\text { RAW } 264.7\end{array}$ & $\begin{array}{l}\text { both cell lines demonstrated high viability } \\
\text { at low NP concentration range }\end{array}$ & {$[19]$} \\
\hline MTS & $\begin{array}{l}\text { NPs composed of PMMA-b- } \\
\text { PMAETMA block copolymer } \\
\text { loaded with bemiparin }\end{array}$ & $\mathrm{BaF} 32$ & $\begin{array}{l}\text { encapsulated bemiparin revealed dose- } \\
\text { dependent increase in cell proliferation }\end{array}$ & {$[10]$} \\
\hline MTS & $\begin{array}{l}\text { Elastin-based NPs loaded with } \\
\text { rapamycin }\end{array}$ & $\begin{array}{l}\text { MDA-MB-468 } \\
\text { MDA-MB-231 }\end{array}$ & $\begin{array}{l}\text { MDA-MB- } 468 \text { cells sensitive to rapamy- } \\
\text { cin revealed more significant decrease in } \\
\text { viability as compared to rapamycin-insen- } \\
\text { sitive MDA-MB-231 cells }\end{array}$ & {$[40]$} \\
\hline SRB & $\begin{array}{l}\text { NPs composed of QPAMN, } \\
\text { adipic and glutaric acid loaded } \\
\text { with paclitaxel }\end{array}$ & $\begin{array}{l}\text { T47D } \\
\text { MDA-MB-231 }\end{array}$ & $\begin{array}{l}\text { both cell lines demonstrated high viability } \\
\text { at low NP concentration range. Cell viabil- } \\
\text { ity was not affected by unloaded NPs }\end{array}$ & {$[34]$} \\
\hline
\end{tabular}

MTS - (3-(4,5-dimethylthiazol-2-yl)-5-(3-carboxymethoxyphenyl)-2-(4-sulfophenyl)-2H-tetrazolium, inner salt, MTS); MTT - 3-(4,5-dimethylthiazol-2-yl)-2,5-diphenyl tetrazolium bromide; NPs - nanoparticles; PLGA - poly(lactide-co-glycolide); PMMA-b-PMAETMA - poly(methyl methacrylate-b-trimethyl aminoethyl methacrylate); QPAMN - N-(1,3-dihydroxypropan-2-yl)-3-methyl-3-(2,4,5-trimethyl-3,6-dioxocyclohexa-1,4-dien-1-yl); SRB - sulforhodamine.

BaF32 - IL-3 dependent and HP sulphate proteoglycan deficient myeloid B cell line stably transfected with recombinant human fibroblast growth factor 2 (FGF) receptor 1c (FGFR-1c); Caco-2 - human colonic carcinoma cell line; HEK293T - human kidney lymphocyte-like cells; HepG2 - human hepatoma cell line; MDA-MB-231 - human breast cancer cells; MDA-MB-468 - human breast cancer cells; RAW 264.7 - mouse macrophage-like cells; SCC7 - squamous carcinoma cells; T47D - human breast cancer cells. 


\section{Polymeric Nanoparticles as Carriers for Therapeutic Agents}

Polymer-based nanoparticles have been successfully used as carriers for antimicrobial agents. For instance, the group of de Carvalho et al. [26] has designed amphotericin-loaded polymeric nanocarriers decorated with maghemite nanoparticles to achieve a site-specific effect to treat cutaneous leishmaniasis. The antibiotic was encapsulated in poly(lactide-co-glycolide) (PLGA) and dimercaptosuccinic acid (DMSA) nanoparticles. The antimicrobial activity of the prepared system was evaluated on C57BL/G mice infected intradermally with promastigotes of Leishmania amazonensis. Remarkably, the controlled drug release from the prepared nanosystem was provided upon exposure to an $\mathrm{AC}$ magnetic field resulting in a magnetohyperthermia effect. The nanocarrier exhibited significantly greater reduction in Leishmania amazonensis number and cell viability as compared to a free form of the antibiotic. Moreover, it allowed an effective reduction of the dose frequency required to obtain the same therapeutic effect [26]. Intriguingly, Chaudhary and Kumar [24] have demonstrated the antibacterial activity of Cefixime-loaded PLGA nanoparticles against the intracellular multidrug resistance (MDR) of Salmonella typhimurium. The results revealed a sustained release of the antibiotic from the prepared formulation and its better permeation across rat intestines as compared to the free drug [24]. A curious instance has been explored by Hill and co-workers [21], who demonstrated that PLGA nanoparticles loaded with the natural antimicrobial cinnamon bark extract are effective inhibitors of Salmonella typhimurium and Listeria monocytogenes. The authors highlighted the potential of the as-prepared system for the food industry to help prevent foodborne diseases [21]. Interesting studies have been conducted by Phan and co-workers [35], who have examined the use of natamycin-loaded nanoparticles composed of dextran and $\operatorname{poly}(\mathrm{D}, \mathrm{L}$-lactide) (Dex-b-PLA) for the surface modification of contact lenses fabricated from various polymeric materials. It has been found that extended drug release up to $12 \mathrm{~h}$ was observed for contact lens materials composed of $\mathrm{N}, \mathrm{N}$-dimethylacrylamide or [tris(trimethylsiloxy)silyl]-propyl methacrylate. The authors suggested that natamycin-loaded Dex-b-PLA nanoparticles incorporated into contact lens materials have the potential to be used for targeted drug delivery directly to the cornea for the treatment of fungal infections [35]. Another group [25] has prepared voriconazole-loaded
PLGA nanoparticles for pulmonary drug delivery. After administration to mice using an inhalation chamber, the sustained drug release was observed for 15 days [25].

Polymeric nanoparticles have also been used successfully as carriers for antihypertensive drugs. As an example, pulmonary delivery of the antihypertensive drug carvedilol was the subject of studies performed by Varshosaz and co-workers [12]. The authors prepared drug-loaded poly(ethyleneco-vinyl acetate) (PEVA) nanoparticles coated with chitosan. The system revealed mucoadhesive properties and provided prolonged drug release up to $8 \mathrm{~h}$. The drug delivery systems based on these nanoparticles spray-dried in the presence of mannitol revealed low density, satisfying flow ability, small aerodynamic diameter and fine powder fraction demonstrating potential as a suitable inhaler powder for the pulmonary delivery of carvedilol [12]. Another group [8] has observed the enhancement of felodipine bioavailability from a formulation based on PLGA nanoparticles. Furthermore, in an ex vivo experiment performed on isolated rat stomach and intestinal segments, the nanovehicles demonstrated sustained release of the antihypertensive drug [8].

Polymer-based nanoparticles have been examined as carriers for other biologically active substances. As an example, Tiwari et al. [29] has exploited the potential of curcumin loaded in PLGA nanoparticles for the induction of neural stem cell (NSC) proliferation in Alzheimer's disease. It has been found that nanoparticles are able to induce a generation of new neurons by internalization into the hippocampus and subventrical zone of adult rats. The authors demonstrated the potential of the prepared system to treat neurodegenerative diseases by enhancing a self-repair mechanism [29]. Polymeric nanoparticles have also been used in the treatment of various dermatoses. As a curious instance, Hussain and co-workers [30] have proposed chitosan nanoparticles co-loaded with hydrocortisone and hydroxytyrosol in the treatment of atopic dermatitis. In this study, hydroxytyrosol was used to decrease the adverse effects of hydrocortisone and provide additional anti-inflammatory and antioxidant properties. The experiment, performed on a NC/Nga mouse model, revealed higher epidermal and dermal accumulation of the as-prepared nanoformulation as compared to a commercial hydrocortisone formulation [30]. Interestingly, PLGA nanoparticles loaded with triamcinolone acetonide have been employed in the treatment of endotoxin-induced uveitis [27]. The authors have evaluated the inflammation mediator expression in a rabbit's eye after intravitreal injection of the endotoxin. The results indicated 
a better performance of triamcinolone-loaded nanoparticles as compared to microparticles of triamcinolone and prednisolone acetate. It has been found that polymeric nanoparticles can be used as an efficient triamcinolone carrier for topical treatment, providing better patient compliance [27]. Alai and Lin [11] have described the application of lansoprazole-loaded polymeric nanoparticles for the treatment of gastric ulcers. The drug encapsulated in Eudragit ${ }^{\circledR}$ RS 100 nanoparticles and PLGA nanoparticles revealed sustained release behavior in vitro. After oral administration of enteric-coated capsules containing the nanoparticles, the sustained release of lansoprazole up to $24 \mathrm{~h}$ was observed in ulcer-induced Wistar rats [11]. Another group [20] has described application of mannose-modified trimethyl chitosan-cysteine conjugate nanoparticles for oral delivery of therapeutic TNF- $\alpha$ siRNA to macrophages. The authors observed effective improvement of siRNA protection in the physiological environment and its enhanced permeation through intestinal epithelium. Furthermore, siRNA uptake by the macrophages was facilitated through clathrin-independent endocytosis. In vitro experiments revealed that orally delivered nanoparticles inhibited TNF- $\alpha$ secretion by macrophages, thus protecting mice with acute hepatic injury from inflammation-induced liver damage [20]. Enteric-coated polymeric nanoparticles have been the subject of studies performed by Hao and co-workers [31]. The authors synthesized core/shell Eudragit ${ }^{\circledR}$ L100-55/Eudragit ${ }^{\circledR}$ RS nanoparticles loaded with aspirin. The external polymer coat provided a $\mathrm{pH}$-sensitive drug release while polymeric core material played the role of the skeleton allowing sustained aspirin release [31].

Other examples demonstrating the application of polymeric nanoparticles as a carrier for various therapeutic agents are listed in Table 3.

\section{Conclusion}

The usage of polymeric nanoparticles as drug carriers for the treatment of many disease entities opens encouraging possibilities in clinical application. The present-day progress achieved in adapting polymeric nanoparticles for drug delivery makes the future of these nanovehicles bright and exciting. Employed as drug carriers, polymerbased nanoparticles offer numerous attractive features such as targeted drug delivery, sustained

Table 3. Use of polymeric nanoparticles as drug vehicles

\begin{tabular}{|c|c|c|c|c|c|}
\hline $\begin{array}{l}\text { Nanoparticle } \\
\text { components }\end{array}$ & $\begin{array}{l}\text { Active } \\
\text { substance }\end{array}$ & $\begin{array}{l}\text { Functionalizing } \\
\text { agent }\end{array}$ & $\begin{array}{l}\text { Cell culture/ } \\
\text { /animal model }\end{array}$ & Pharmacological effect & Reference \\
\hline $\begin{array}{l}\text { PLA-TPGS, } \\
\text { TPGS-COOH }\end{array}$ & docetaxel & herceptin & SK-BR-3 & targeted delivery of anticancer drugs & {$[13]$} \\
\hline PLGA & docetaxel & $\begin{array}{l}\text { bombesin pep- } \\
\text { tide }\end{array}$ & MDA-MB-231 & $\begin{array}{l}\text { targeted delivery of anticancer drugs. } \\
\text { Sustained drug release }\end{array}$ & [7] \\
\hline $\begin{array}{l}\text { Carboxymethyl } \\
\text { dextran }\end{array}$ & DOX & $\begin{array}{l}\text { 2-nitrozoimid- } \\
\text { azole derivative }\end{array}$ & SCC7 & $\begin{array}{l}\text { hypoxia-responsive and sustained } \\
\text { drug release }\end{array}$ & [15] \\
\hline $\begin{array}{l}\text { PMMA, biosur- } \\
\text { factants (rham- } \\
\text { nolipid, surfactin, } \\
\text { trehalose) }\end{array}$ & $\begin{array}{l}\text { ibuprofen, } \\
\text { anthraqui- } \\
\text { none, cur- } \\
\text { cumin }\end{array}$ & - & PMBCs & $\begin{array}{l}\text { PH-responsive and sustained drug } \\
\text { release; antibacterial activity against } \\
\text { Bacillus subtilis and Pseudomonas aeru- } \\
\text { ginosa by induction of oxidative stress }\end{array}$ & [36] \\
\hline Chitosan & dorzolamide & - & $\begin{array}{l}\text { Goat cornea, } \\
\text { male albino } \\
\text { rabbit }\end{array}$ & $\begin{array}{l}\text { reduced frequency of administration } \\
\text { in the treatment of glaucoma }\end{array}$ & [9] \\
\hline POX & $\begin{array}{l}\text { hydroxyben- } \\
\text { zyl alcohol }\end{array}$ & - & $\begin{array}{l}\text { RAW 264.7/ } \\
\text { Balb/c mice }\end{array}$ & $\begin{array}{l}\text { simultaneous antioxidant and anti- } \\
\text { inflammatory effect }\end{array}$ & {$[22]$} \\
\hline $\mathrm{PVO}$ & - & - & $\begin{array}{l}\text { RAW 264.7/ } \\
\text { Balb/c mice }\end{array}$ & $\begin{array}{l}\text { step-wise release of antioxidant and } \\
\text { anti-inflammatory vanillin upon hydro- } \\
\text { lytic degradation of polymeric matrix }\end{array}$ & [39] \\
\hline
\end{tabular}

DOX - doxorubicin; PLA-TPGS - poly(lactide)-D-a-tocopheryl polyethylene glycol 1000 succinate copolymer;

PLGA - poly(lactide-co-glycolide); PMMA - poly(methyl methacrylate); POX - polyoxalate; PVO - poly(vanillin oxalate); TPGS-COOH - carboxyl group-terminated D- $\alpha$-tocopheryl polyethylene glycol 1000 succinate.

MDA-MB-231 - gastrin releasing peptide receptor positive breast cancer cells; PBMCs - peripheral blood mononuclear cells; RAW 264.7 - mouse macrophage-like cells; SCC7 - squamous carcinoma cells; SK-BR-3 - breast cancer cells. 
release or prolonged circulation half-life. Polymeric nanoparticles can protect the drug from degradation in physiological conditions and improve its bioavailability. Polymeric nanoparticle-based drug delivery systems have been successfully employed as carriers for anticancer, anti-inflammatory and antihypertensive agents, antioxidants, and nucleic acids, among others. In spite of the scientific advances in the usage of polymeric nanoparticles as drug vehicles, many more efforts from scientists from a variety of disciplines are needed before they can be fully exploited for therapeutic applications.

\section{References}

[1] Moritz M, Geszke-Moritz M: Application of nanomaterials in medical sciences. Chemik 2012, 66, 219-226.

[2] Geszke-Moritz M, Moritz M: Quantum dots as versatile probes in medical sciences: Synthesis, modification and properties. Mater Sci Eng C 2013, 33, 1008-1021.

[3] Yang K, Feng L, Shi X, Liu Z: Nano-graphene in biomedicine: theranostic applications. Chem Soc Rev 2013, 42, 530-547.

[4] Battigelli A, Ménard-Moyon C, Ros TD, Prato M, Bianco A: Endowing carbon nanotubes with biological and biomedical properties by chemical modifications. Adv Drug Delivery Rev 2013, 65, 1899-1920.

[5] Moritz M, Geszke-Moritz M: The newest achievement in synthesis, immobilization and practical applications of antibacterial nanoparticles. Chem Eng J 2013, 228, 596-613.

[6] Han S, Li M, Liu X, Gao H, Wu Y: Construction of amphiphilic copolymer nanoparticles based on gelatin as drug carriers for doxorubicin delivery. Colloids Surf B 2013, 102, 833-841.

[7] Kulhari H, Pooja D, Shrivastava S, Naidu VGM, Sistla R: Peptide conjugated polymeric nanoparticles as a carrier for targeted delivery of docetaxel. Colloids Surf B 2014, 117, 166-173.

[8] Shah U, Joshi G, Sawant K: Improvement in antihypertensive and antianginal effects of felodipine by enhanced absorption from PLGA nanoparticles optimized by factorial design. Mater Sci Eng C 2014, 35, 153-163.

[9] Katiyar S, Pandit J, Mondal RS, Mishra AK, Chuttani K, Aqil M, Ali A, Sultana Y: In situ gelling dorzolamide loaded chitosan nanoparticles for the treatment of glaucoma. Carbohydr Polym 2014, 102, 117-124.

[10] Reyes-Ortega F, Rodríguez G, Aguilar MR, Lord M, Whitelock J, Stenzel MH, Román JS: Encapsulation of low molecular weight heparin (bemiparin) into polymeric nanoparticles obtained from cationic block copolymers: properties and cell activity. J Mater Chem B 2013, 1, 850-860.

[11] Alai M, Lin WJ: Novel lansoprazole-loaded nanoparticles for the treatment of gastric acid secretion-related ulcers: In vitro and in vivo pharmacokinetic pharmacodynamic evaluation. Amer Assoc Pharm Sci J 2014, DOI: 10.1208/ /s12248-014-9564-0.

[12] Varshosaz J, Taymouri S, Hamishehkar H: Fabrication of polymeric nanoparticles of poly(ethylene-co-vinyl acetate) coated with chitosan for pulmonary delivery of carvedilol. J Appl Polym Sci 2014, 131, 39694-39701.

[13] Zhao J, Feng SS: Effects of PEG tethering chain length of vitamin E TPGS with a Herceptin-functionalized nanoparticle formulation for targeted delivery of anticancer drugs. Biomaterials 2014, 35, 3340-3347.

[14] Pu HL, Chiang WL, Maiti B, Liao ZX, Ho YC, Shim MS, Chuang EY, Xia Y, Sung HW: Nanoparticles with dual responses to oxidative stress and reduced $\mathrm{pH}$ for drug release and anti-inflammatory applications. ACS Nano 2014, $8,1213-1221$.

[15] Thambi T, Deepagan VG, Yoon HY, Han HS, Kim SH, Son S, Jo DG, Ahn CH, Suh YD, Kim K, Kwon IC, Lee DS, Park JH: Hypoxia-responsive polymeric nanoparticles for tumor-targeted drug delivery. Biomaterials 2014, 35, 1735-1743.

[16] Zhang X, Chen G, Wen L, Yang F, Shao A, Li X, Long W, Mu L: Novel multiple agents loaded PLGA nanoparticles for brain delivery via inner ear administration: In vitro and in vivo evaluation. Eur J Pharm Sci 2013, 48, 595-603.

[17] Zhang X, Dong Y, Zeng X, Liang X, Li X, Tao W, Chen H, Jiang Y, Mei L, Feng SS: The effect of autophagy inhibitors on drug delivery using biodegradable polymer nanoparticles in cancer treatment. Biomaterials 2014, 35, 1932-1943.

[18] Narayanan D, Geena MG, Lakshmi H, Manzoor K, Shantikumar N, Menon D: Poly-(ethylene glycol) modified gelatin nanoparticles for sustained delivery of the anti-inflammatory drug ibuprofen-sodium: An in vitro and in vivo analysis. Nanomedicine: NBM 2013, 9, 818-828.

[19] Forbes DC, Peppas NA: Polycationic nanoparticles for siRNA delivery: Comparing ARGET ATRP and UV-initiated formulations. ACS Nano 2014, 8, 2908-2917.

[20] He C, Yin L, Tang C, Yin C: Multifunctional polymeric nanoparticles for oral delivery of TNF-a siRNA to macrophages. Biomaterials 2013, 34, 2843-2854.

[21] Hill LE, Taylor TM, Gomes C: Antimicrobial efficacy of poly(DL-lactide-co-glycolide) (PLGA) nanoparticles with entrapped cinnamon bark extract against Listeria monocytogenes and Salmonella typhimurium. J Food Sci 2013, $78,626-632$.

[22] Yoo D, Guk K, Kim H, Khang G, Wu D, Lee D: Antioxidant polymeric nanoparticles as novel therapeutics for airway inflammatory diseases. Int J Pharm 2013, 450, 87-94.

[23] Zakeri-Milani P, Loveymi BD, Jelvehgari M, Valizadeh H: The characteristics and improved intestinal permeability of vancomycin PLGA-nanoparticles as colloidal drug delivery system. Colloids Surf B 2013, 103, $174-181$.

[24] Chaudhary SH, Kumar V: Taguchi design for optimization and development of antibacterial drug-loaded PLGA nanoparticles. Int J Biol Macromol 2014, 64, 99-105. 
[25] Sinha B, Mukherjee B, Pattnaik G: Poly-lactide-co-glycolide nanoparticles containing voriconazole for pulmonary delivery: in vitro and in vivo study. Nanomedicine: NBM 2013, 9, 94-104.

[26] de Carvalho RF, Ribeiro IF, Miranda-Vilela AL, Filho J de Sousa, Martins OP, Cintra e Silva Dde O, Tedesco AC, Lacava ZGM, Báo SN, Sampaio RNR: Leishmanicidal activity of amphotericin B encapsulated in PLGADMSA nanoparticles to treat cutaneous leishmaniasis in C57BL/6 mice. Exp Parasitol 2013, 135, 217-222.

[27] Sabzevari A, Adibkia K, Hashemi H, Hedayatfar A, Mohsenzadeh N, Atyabi F, Ghahremani MH, Dinarvand R: Polymeric triamcinolone acetonide nanoparticles as a new alternative in the treatment of uveitis: In vitro and in vivo studies. Eur J Pharm Biopharm 2013, 84, 63-71.

[28] Palange AL, Mascolo DD, Carallo C, Gnasso A, Decuzzi P: Lipid-polymer nanoparticles encapsulating curcumin for modulating the vascular deposition of breast cancer cells. Nanomedicine: NBM 2014, DOI: 10.1016/j. nano.2014.02.004.

[29] Tiwari SK, Agarwal S, Seth B, Yadav A, Nair S, Bhatnagar P, Karmakar M, Kumari M, Chauhan LKS, Patel DK, Srivastava V, Singh D, Gupta SK, Tripathi A, Chaturvedi RK, Gupta KC: Curcumin-loaded nanoparticles potently induce adult neurogenesis and reverse cognitive deficits in Alzheimer's disease model via canonical W nt/ $\beta$-catenin pathway. ACS Nano 2014, 8, 76-103.

[30] Hussain Z, Katas H, Amin MCIM, Kumolosasi E, Buang F, Sahudin S: Self-assembled polymeric nanoparticles for percutaneous co-delivery of hydrocortisone/hydroxytyrosol: An ex vivo and in vivo study using an NC/Nga mouse model. Int J Pharm 2013, 444, 109-119.

[31] Hao S, Wang B, Wang Y, Xu Y: Enteric-coated sustained-release nanoparticles by coaxial electrospray: preparation, characterization, and in vitro evaluation. J Nanopart Res 2014, 16, 2204-2214.

[32] Jana S, Maji N, Nayak AK, Sen KK, Basu SK: Development of chitosan-based nanoparticles through inter-polymeric complexation for oral drug delivery. Carbohydr Polym 2013, 98, 870-876.

[33] Dionísio M, Cordeiro C, Remuñán-López C, Seijo B, da Costa AMR, Grenha A: Pullulan-based nanoparticles as carriers for transmucosal protein delivery. Eur J Pharm Sci 2013, 50, 102-113.

[34] Bae J, Nael MA, Jiang L, Hwang PT, Mahdi F, Jun H-W, Elshamy WM, Zhou YD, Murthy SN, Doerksen RJ, Jo S: Quinone propionic acid-based redox-triggered polymer nanoparticles for drug delivery: Computational analysis and in vitro evaluation. J Appl Polym Sci 2014, 131, 40461-40470.

[35] Phan CM, Subbaraman L, Liu S, Gu F, Jones L: In vitro uptake and release of natamycin Dex-b-PLA nanoparticles from model contact lens materials. J Biomater Sci, Polym Ed 2014, 25, 18-31.

[36] Hazra C, Kundu D, Chatterjee A, Chaudhari A, Mishra S: Poly(methyl methacrylate) (core)-biosurfactant (shell) nanoparticles: Size controlled sub-100 nm synthesis, characterization, antibacterial activity, cytotoxicity and sustained drug release behavior. Colloids Surf A 2014, 449, 96-113.

[37] Stevanović M, Bračko I, Milenković M, Filipović N, Nunić J, Filipič M, Uskoković DP: Multifunctional PLGA particles containing poly(L-glutamic acid)-capped silver nanoparticles and ascorbic acid with simultaneous antioxidative and prolonged antimicrobial activity. Acta Biomater 2014, 10, 151-162.

[38] Papa S, Ferrari R, de Paola M, Rossi F, Mariani A, Caron I, Sammali E, Peviani M, Dell'Oro V, Colombo C, Morbidelli M, Forloni G, Perale G, Moscatelli D, Veglianese P: Polymeric nanoparticle system to target activated microglia/macrophages in spinal cord injury. J Controlled Release 2014, 174, 15-26.

[39] Kwon J, Kim J, Park S, Khang G, Kang PM, Lee D: Inflammation-responsive antioxidant nanoparticles based on a polymeric prodrug of vanillin. Macromolecules 2013, 14, 1618-1626.

[40] Shi P, Aluri S, Lin Y-A, Shah M, Edman M, Dhandhukia J, Cui H, MacKay JA: Elastin-based protein polymer nanoparticles carrying drug at both corona and core suppress tumor growth in vivo. J Controlled Release 2013, $171,330-338$.

\section{Address for correspondence:}

Michał Moritz

Faculty of Chemical Technology

Institute of Chemistry and Technical Electrochemistry

Poznan University of Technology

Berdychowo 4

60-965 Poznań

Poland

Tel. +48 616652316

E-mail: michal.moritz@put.poznan.pl

Conflict of interest: None declared

Received: 27.04.2014

Revised: 25.09.2014

Accepted: 6.10.2014 